Supporting Information

\title{
Resistive Switching Effect of Multiferroic Complex Oxide Solid Solution Thin Films
}

Fang $\mathrm{Hu}^{1,2}$, Wenjie Ming ${ }^{1,2}$, Liu Yang ${ }^{1,3}$, Can Huang ${ }^{4}$, Hongyang Zhao ${ }^{5}$, Shuhong $\mathrm{Xie}^{2}$, Zhenxiang Cheng ${ }^{6^{*}}$, Tingting Jia ${ }^{1, *}$

1 Shenzhen Institute of Advanced Electronic Materials, Shenzhen Institutes of Advanced Technology, Chinese Academy of Sciences, Shenzhen 518055, China

${ }^{2}$ School of Materials Science and Engineering, Xiangtan University, Xiangtan 411105, China

${ }^{3}$ College of Physics, Qingdao University, Qingdao 266071, China

${ }^{4}$ Faculty of Materials Science and Chemistry, China University of Geosciences, Wuhan, Hubei 430074, China

${ }^{5}$ Hubei Key Laboratory of Plasma Chemistry and Advanced Materials, Department of Materials Science and Engineering, Wuhan Institute of Technology, Wuhan 430205, China

${ }^{6}$ Institute for Superconducting \& Electronic Materials, University of Wollongong, Innovation Campus, North Wollongong, NSW 2500, Australia
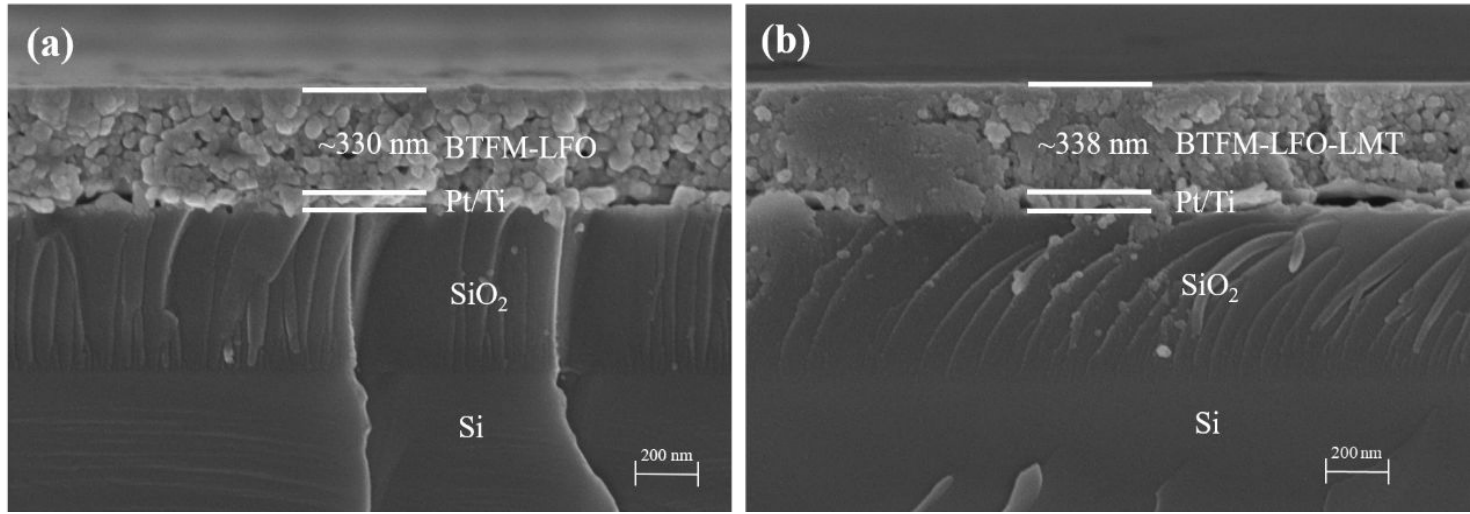

Figure S1 SEM images of cross section from a BTFM-LFO and b BTFM-LFO-LMT film.

*Corresponding author: tt.jia@siat.ac.cn (Tingting Jia), cheng@uow.edu.au (Zhenxiang Cheng) 

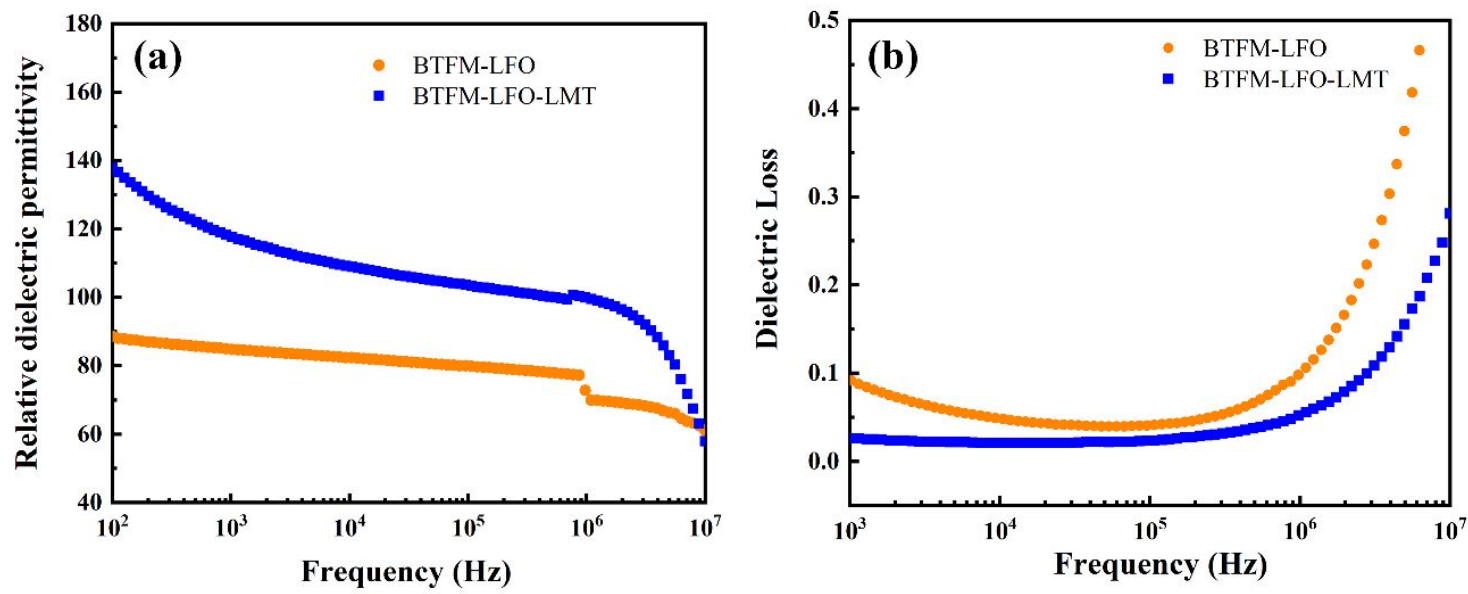

Figure S2 a Frequency dependence of the relative dielectric permittivity, and $\mathbf{b}$ dielectric loss for the BTFM-LFO and BTFM-LFO-LMT films. 